\title{
UNIDADES DE TERAPIA INTENSIVA NO BRASIL E A FILA ÚNICA DE LEITOS NA PANDEMIA DE COVID-19
}

Maria Clara Marques Fagundes ${ }^{1}$

Neyson Pinheiro Freire ${ }^{2}$

Maria Helena Machado ${ }^{3}$

Francisco Rosemiro Guimarães Ximenes Neto ${ }^{34}$ https://orcid.org/0000-0003-4050-5655

https://orcid.org/0000-0002-9038-9974

https://orcid.org/0000-0002-5209-2424

https://orcid.org/0000-0002-7905-9990

Objetivo: Discutir a proposta de Fila Única nas Unidades de Terapia Intensiva (UTI) durante a pandemia de COVID-19. Método: Pesquisa quantitativa, descritiva, desenvolvida durante os meses de abril a junho de 2020 , com buscas de dados em sites oficiais e relatórios institucionais. Resultados: No Brasil, apenas 5,3\% dos municipios possuem leitos de UTI. Os estados da região Norte apresentam os menores indicadores de leitos/100 mil habitantes. O Sistema Único de Saúde detém 54,0\% dos leitos do país. Nos estados do Rio de Janeiro, Mato Grosso, São Paulo e no Distrito Federal os leitos não SUS predominam. A região Sudeste possui o maior cluster (50,2\%) de leitos. Dos 8.980 leitos de UTI COVID-19, $53,8 \%$ estão sob a gestão municipal e $46,2 \%$ estadual; $37,9 \%$ estão na região Sudeste e $29,5 \%$ na Nordeste. Conclusões: O colapso do sistema de saúde, projetado por especialistas frente ao avanço da COVID-19, já chegou a alguns estados. A desigualdade no número de leitos per capita entre as redes públicas e privadas dificulta a racionalização dos recursos, sendo observadas também disparidades regionais. A criação de uma "fila única" nas UTI é uma medida necessária para promover um acesso mais equitativo e racionalizar decisões.

Descritores: Sistema Único de Saúde; Unidades de Terapia Intensiva; Pandemias; Infecções por Coronavirus; Acesso aos Serviços de Saúde.

\section{INTENSIVE CARE UNITS IN BRAZIL AND THE SINGLE QUEUE OF BEDS IN THE COVID-19 PANDEMIC}

Objective: To discuss the proposal for a Single Queue in Intensive Care Units (ICU) during COVID-19 pandemic in Brazil. Method: Quantitative, descriptive research, carried out from April to June 2020, with data searches on official websites and institutional reports. Results: In Brazil, only $5.3 \%$ of municipalities have ICU beds. The states of North region have lowest bed indicators $/ 100$ thousand inhabitants. Health Unic System holds 54.0\% of the country's beds. In states of Rio de Janeiro, Mato Grosso, São Paulo and Federal District, non-HUS beds predominate. The Southeast region has largest cluster (50.2\%) of beds. Of 8,980 ICU beds COVID-19, 53.8\% are under municipal management and $46.2 \%$ state $37.9 \%$ are in Southeast and $29.5 \%$ in Northeast. Conclusions: The collapse of health system, designed by specialists in face of advancement of COVID-19, has already reached some states. The inequality in number of beds per capita between public and private networks makes it difficult to rationalize resources, and regional disparities are also observed. The creation of a "single row" in ICU is a necessary measure to promote more equitable access and to rationalize decisions.

Descriptors: Unified Health System; Intensive Care Units; Pandemics; Coronavirus Infections; Health Services Accessibility.

\section{UNIDADES DE CUIDADO INTENSIVO EN BRASIL Y LA SOLA COLA DE CAMAS EN LA PANDEMIA COVID-19}

Objetivo: Discutir la propuesta de una Cola Única en Unidades de Cuidados Intensivos (UCI) durante la pandemia de COVID-19 en Brasil. Método: Investigación cuantitativa, descriptiva, realizada de abril a junio de 2020, con búsquedas de datos en sitios web oficiales e informes institucionales. Resultados: En Brasil, solo 5,3\% de los municipios tienen camas en UCI. Los estados de la región Norte tienen los indicadores de camas más bajos/100 mil habitantes. Sistema Único de Salud posee el 54.0\% de las camas del país. En los estados de Río de Janeiro, Mato Grosso, São Paulo y Distrito Federal, predominan las camas que no son del SUS. La región Sudeste tiene el grupo más grande (50,2\%) de camas. De las 8,980 camas de UCI COVID-19, $53.8 \%$ están bajo administración municipal y $46.2 \%$ estatales; $37.9 \%$ están en Sudeste y $29.5 \%$ en Noreste. Conclusiones: El colapso del sistema de salud, diseñado por especialistas ante el avance de COVID-19, ya ha llegado a algunos estados. La desigualdad en número de camas per cápita entre las redes públicas y privadas dificulta la racionalización de los recursos, y también se observan disparidades regionales. La creación de una "fila única" en las UCI es una medida necesaria para promover un acceso más equitativo y racionalizar las decisiones.

Descriptores: Sistema Único de Salud; Unidades de Cuidados Intensivos; Pandemias; Infecciones por Coronavirus; Accesibilidad a los Servicios de Salud.

IUniversidade Federal Fluminense (UFF). Grupo de Pesquisa Maternidade, Saúde da Mulher e da Criança da Niterói-RJ, Brasil.

${ }^{2}$ Universidade Federal de São Paulo (Unifesp). Grupo de Estudos e Pesquisa em Administração dos Serviços de Saúde e Gerenciamento de Enfermagem - GEPAG/EPE/ São Paulo-SP, Brasil.

${ }^{3}$ Escola Nacional de Saúde Pública Sérgio Arouca. Fiocruz - Rio de Janeiro-RJ, Brasil.

${ }^{4}$ Universidade Estadual Vale do Acaraú (UVA), Sobral, CE, Brasil.

Autor Correspondente: Neyson Pinheiro Freire. E-mail: neysonfreire@gmail.com

Recebido: 7/7/20 Aceito: 17/7/20 


\section{INTRODUÇÃO}

Em dezembro de 2019, a China anunciou uma série de casos de pneumonia atípica, com apresentação clínica semelhante à doença viral que, posteriormente, descobriu-se que era causada por um novo coronavírus, o SARS-CoV-2. A doença causada pelo vírus foi denominada COVID-19 e despertou atenção internacionall-3.

Com o avanço dos casos e óbitos, em 30 de janeiro de 2020, a Organização Mundial de Saúde (OMS), declarou o surto causado pelo SARS-CoV-2 uma "Emergência de Saúde Pública de Importância Internacional (ESPII)", o mais alto nivel de alerta, conforme previsto no Regulamento Sanitário Internacional ${ }^{4}$.

Com a expansão do número de casos e óbitos e a disseminação da doença para 114 países, em 11 de março de 2020, a OMS caracterizou a situação da COVID-19 como pandemia ${ }^{5}$. 0 primeiro caso confirmado na América Latina foi o reportado no Brasil, em 26 de fevereiro, na cidade de São Paulo, em um homem de 61 anos, que havia retornado de viagem para a Itália .

A amplitude e magnitude da COVID-19, sua expansão para as diversas nações e territórios e a elevada letalidade dos casos graves, demandou dos governos ações e estratégias rápidas, relativas a insumos e equipamentos, para suprir as necessidades dos serviços de saúde, bem como redefinir o papel dos sistemas de saúde no momento de crise mundial pandêmica. Mais do que nunca, a Atenção Primária à Saúde (APS) teve que assumir seu papel de coordenadora do cuidado e ordenadora das Redes de Atenção à Saúde (RAS), assim como a vigilância do território, e efetuar o manejo clínico dos casos positivos assintomáticos e leves. A média e a alta complexidade passaram por um reordenamento, com importante arranjo organizacional, para acolher os casos que demandam cuidados clínicos especializados, com maior suporte profissional e tecnológico, em nivel hospitalar, sobretudo nas Unidades de Terapia Intensiva (UTI), que logo acenderam o alerta de colapso.

Nesse contexto, este estudo objetiva discutir a proposta da Fila Única nas UTI durante a pandemia de COVID-19 no Brasil.

\section{MÉTODO}

\section{Tipo de Estudo}

Pesquisa quantitativa, descritiva, com dados secundários sobre a situação dos leitos de UTI e a pandemia de COVID-19 no Brasil.

\section{LOCAL DO ESTUDO}

O estudo teve abrangência nacional e foi desenvolvido com dados de todos os estados brasileiros e do Distrito Federal.

\section{PARTICIPANTES DO ESTUDO}

Foram incluidos unidades e serviços de saúde públicos e privados, que prestam atendimento a pacientes com COVID-19, em todo o território nacional. Foram excluidos serviços e unidades de saúde que apresentaram dificuldade de acesso aos dados ou que não possuíam informações atualizadas.

\section{COLETA DE DADOS}

Os dados foram coletados durante o período de abril a junho de 2020 de sites, redes sociais oficiais e relatórios do Ministério da Saúde, das Secretarias Estaduais de Saúde, Conselho Nacional de Secretários da Saúde (CONASS), Fundação Oswaldo Cruz (FIOCRUZ), Instituto Brasileiro de Geografia e Estatística (IBGE), dentre outras instituições, que congregam dados gerais do país e por estado, atualizados e de acesso público, sobre o número de casos, óbitos, leitos de UTI e leitos habilitados SRAG/UTI COVID-19.

\section{PROCEDIMENTOS DE ANÁLISE E TRATAMENTO DOS DADOS}

Os dados foram sistematizados em uma planilha do programa Excel@ e efetuada a análise descritiva, considerando as frequências e porcentagens das variáveis, além da média, mediana e do desvio padrão.

\section{ASPECTOS ÉTICOS}

Por utilizar dados secundários, de acesso aberto, o estudo dispensa avaliação do Comitê de Ética em Pesquisa. Contudo, buscou-se respeitar os princípios ético-legais emanados pela Resolução no 466/2012, do Conselho Nacional de Saúde (CNS) ${ }^{7}$.

\section{RESULTADOS}

Até o mês de junho de 2020, as regiões brasileiras mais atingidas pela COVID-19 foram: Norte, especialmente os estados do Amazonas que, entre abril e junho, passou de 8.109 para 58.018 casos e de 649 para 2.549 óbitos, e o Pará, que foi de 4.472 para 71.243 casos e de 369 óbitos para 4.291 óbitos; a região Nordeste, onde despontam quatro dos nove estados - Maranhão: de 4.530 para 62.711 casos e de 271 para 1.537 óbitos; Ceará: de 11.470 para 81.289 casos e de 795 para 5.070 óbitos; Pernambuco: de 9.325 para 46.427 casos e de 749 para 3.959 óbitos; e Bahia: de 4.040 para 39.206 casos e de 146 óbitos para 1.181 óbitos; e a Região Sudeste, tendo São Paulo como epicentro da 
pandemia no Brasil, onde foi registrado um salto de 34.053 para 190.285 casos e de 2.851 para 11.132 óbitos; e Rio de Janeiro, que passou de 12.391 para 83.343 casos e de 1.123 para 7.967 óbitos por COVID-198

Em relação à disponibilidade de leitos de UTI para o atendimento dos casos graves de COVID-19, dados da Fundação Osvaldo Cruz (FIOCRUZ) apontam a indisponi- bilidade de leitos de UTI em municípios com menos de 20 mil habitantes. Além disso, pelo menos 50\% dos municípios com até 100 mil habitantes, possuem taxa de "zero" leitos de UTI por 100 mil habitantes. As maiores taxas de leitos de UTI disponíveis ao SUS, por 100 mil habitantes estão em municípios com 100 a 500 mil ou mais de 500 mil habitantes (Tabela 1).

Tabela 1 - Taxa de leitos de UTI disponíveis ao SUS por 100 mil habitantes segundo contingente populacional. Brasil, 2020.

\begin{tabular}{|l|c|c|c|c|c|}
\hline \multicolumn{1}{|c|}{ Classe populacional } & Valor mínimo & Valor Máximo & Média & Desvio Padrão & Mediana \\
\hline Até 20 mil & 0,0 & 0,0 & 0,0 & 0,0 & 0,0 \\
\hline 20 a 50 mil & 0,0 & 41,8 & 1,9 & 6,7 & 0,0 \\
\hline 50 a 100 mil & 0,0 & 21,9 & 3,2 & 5,5 & 0,0 \\
\hline 100 a 500 mil & 0,0 & 42,2 & 5,8 & 5,6 & 5,8 \\
\hline Mais de 500 mil & 1,3 & 18,8 & 8,0 & 3,6 & \\
\hline
\end{tabular}

Fonte: Fundação Osvaldo Cruz, MonitoraCovid-199.

Conforme mostra a Tabela 2, a distribuição do número de leitos de UTI por 100 mil habitantes nos estados brasileiros é bastante desigual. Os menores índices estão na região Norte (Roraima: 4 leitos/100 habitantes; Amapá e Acre: 5 leitos/100 habitantes; Amazonas: 7 leitos/100 habitantes; Pará:
8 leitos/100 habitantes). Os maiores índices estão na região Sudeste (São Paulo: 19 leitos/100 habitantes; Espírito Santo: 20 leitos/100 habitantes; Rio de Janeiro: 25 leitos/100 habitantes). O Distrito Federal registra o maior indicador (30 leitos/100 habitantes) do território nacional.

Tabela 2 - Unidades federadas e número de leitos de UTI a cada 100 mil habitantes, em ordem crescente.

\begin{tabular}{|c|c|c|}
\hline Unidade da Federação & População Total & Leitos de UTI/100 mil habitantes* \\
\hline Roraima & 605.761 & 4 \\
\hline Amapá & 845.731 & 5 \\
\hline Acre & 881.935 & 5 \\
\hline Amazonas & 4.144 .597 & 7 \\
\hline Piauí & 3.273 .227 & 7 \\
\hline Tocantins & 1.572 .866 & 8 \\
\hline Maranhão & 7.075 .181 & 8 \\
\hline Pará & 8.602 .865 & 8 \\
\hline Alagoas & 3.337 .357 & 9 \\
\hline Ceará & 9.132 .078 & 9 \\
\hline Bahia & 14.873 .064 & 10 \\
\hline Sergipe & 2.298 .696 & 10 \\
\hline Santa Catarina & 7.164.788 & 12 \\
\hline Rio Grande do Norte & 3.506 .853 & 12 \\
\hline Paraíba & 4.018 .127 & 12 \\
\hline Rondônia & 1.777 .225 & 13 \\
\hline Mato Grosso do Sul & 2.778 .986 & 14 \\
\hline Rio Grande do Sul & 11.377 .239 & 14 \\
\hline Goiás & 7.018 .354 & 15 \\
\hline Minas Gerais & 21.168 .791 & 15 \\
\hline Pernambuco & 9.557.071 & 16 \\
\hline Mato Grosso & 3.484 .466 & 17 \\
\hline Paraná & 11.433 .957 & 18 \\
\hline São Paulo & 45.919 .049 & 19 \\
\hline Espírito Santo & 4.018 .650 & 20 \\
\hline Rio de Janeiro & 17.264 .943 & 25 \\
\hline Distrito Federal & 3.015 .268 & 30 \\
\hline
\end{tabular}

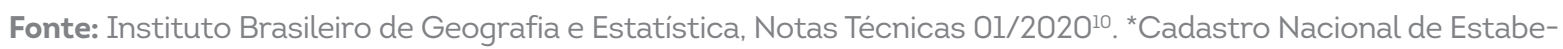
lecimentos de Saúde - CNES/DATASUS, dados de dezembro de 2019. 
A Tabela 3 apresenta os dados da disponibilidade de leitos de UTI no Brasil, SUS e não SUS. Embora 54,0\% dos leitos de UTI do país pertençam ao SUS, a disponibilização de leitos pela iniciativa privada/filantrópica (redes de hospitais, planos de saúde/saúde suplementar) é predominante nos estados do Rio de Janeiro (68,9\%), Mato Grosso (64,8\%), São Paulo $(51,7 \%)$ e no Distrito Federal (73,8\%).
Independente da capacidade instalada de serviços de saúde, todos os estados brasileiros possuem leitos de UTI SUS e não SUS. Contudo, a região Sudeste apresenta a maior concentração do número de leitos (50,2\%). Enquanto a rede hospitalar da região Norte possui apenas 5,0\% dos leitos, o estado de São Paulo detém sozinho, 27,0\% do total de leitos do país.

Tabela 3 - Disponibilidade de leitos de UTI no Brasil, 2020.

\begin{tabular}{|c|c|c|c|}
\hline Unidades da Federação & sus & Não SUS & Total \\
\hline Brasil & 18.564 & 15.754 & 34.318 \\
\hline Região Norte & 1.182 & 536 & 1.718 \\
\hline Acre & 43 & 15 & 58 \\
\hline Amapá & 44 & 34 & 78 \\
\hline Amazonas & 356 & 94 & 450 \\
\hline Pará & 400 & 261 & 661 \\
\hline Rondônia & 213 & 72 & 285 \\
\hline Roraima & 17 & 8 & 25 \\
\hline Tocantins & 109 & 52 & 161 \\
\hline Região Nordeste & 4.337 & 2.643 & 6.980 \\
\hline Alagoas & 315 & 111 & 426 \\
\hline Bahia & 869 & 704 & 1.573 \\
\hline Ceará & 630 & 372 & 1.002 \\
\hline Maranhão & 413 & 269 & 682 \\
\hline Paraiba & 372 & 164 & 536 \\
\hline Pernambuco & 1.026 & 621 & 1.647 \\
\hline Piauí & 206 & 87 & 293 \\
\hline Rio Grande do Norte & 306 & 220 & 526 \\
\hline Sergipe & 200 & 95 & 295 \\
\hline Região Sudeste & 8.201 & 9.031 & 17.232 \\
\hline Espírito Santo & 406 & 398 & 804 \\
\hline Minas Gerais & 2.068 & 1.083 & 3.151 \\
\hline Rio de Janeiro & 1.247 & 2.761 & 4.008 \\
\hline São Paulo & 4.480 & 4.789 & 9.269 \\
\hline Região Sul & 3.431 & 1.737 & 5.168 \\
\hline Paraná & 1.656 & 788 & 2.444 \\
\hline Rio Grande do Sul & 1.015 & 645 & 1.660 \\
\hline Santa Catarina & 760 & 304 & 1.064 \\
\hline Região Centro-Oeste & 1.413 & 1.807 & 3.220 \\
\hline Distrito Federal & 261 & 736 & 997 \\
\hline Goiás & 675 & 525 & 1.200 \\
\hline Mato Grosso & 208 & 383 & 591 \\
\hline Mato Grosso do Sul & 269 & 163 & 432 \\
\hline
\end{tabular}

Fonte: Ministério da Saúde, Painel de leitos e insumos ${ }^{11}$. 
A Tabela 4 descreve os leitos adultos e pediátricos habilitados SRAG/UTI COVID-19. No Brasil, há apenas 297 municípios com leitos de UTI, que correspondem $5,3 \%$ dos 5.570 municipios brasileiros. O maior número de municípios com leitos de UTI encontra-se nas regiões Sudeste (92), Nordeste (80) e Sul (78), e nos estados do Rio Grande do Sul (38), São Paulo (37), Minas Gerais (24), Rio de Janeiro (24) e Santa Catarina (22).
No período de 15 de abril e 26 de junho, foram habilitados 8.980 leitos para COVID-19, no Brasil, sendo 8.744 (97,4\%) para adultos. A maioria destes leitos (53,8\%) estão sob gestão municipal e os demais, sob gestão estadual. Quanto à distribuição espacial, a maioria dos leitos está na Sudeste (37,9\%) habilitou mais leitos (3.382) do que as regiões Norte, Sul e Centro-Oeste juntas (2.797 leitos).

Tabela 4 - Leitos habilitados SRAG/UTI COVID-19. Brasil, 2020.

\begin{tabular}{|c|c|c|c|c|}
\hline Unidades da Federação & $\begin{array}{c}\text { Municipios com } \\
\text { Leitos }\end{array}$ & Leitos Adultos & Leitos Pediátricos & Leitos Total \\
\hline Região Norte & 21 & 690 & 40 & 730 \\
\hline Acre & 2 & 30 & 0 & 30 \\
\hline Amazonas & 1 & 186 & 8 & 194 \\
\hline Pará & 9 & 322 & 14 & 336 \\
\hline Rondônia & 5 & 74 & 7 & 81 \\
\hline Roraima & 1 & 10 & 5 & 15 \\
\hline Tocantins & 2 & 36 & 6 & 42 \\
\hline Ceará & 13 & 381 & 21 & 402 \\
\hline Maranhão & 9 & 238 & 0 & 238 \\
\hline Paraíba & 6 & 170 & 0 & 170 \\
\hline Pernambuco & 14 & 619 & 25 & 644 \\
\hline Piauí & 7 & 281 & 10 & 291 \\
\hline Rio Grande do Norte & 9 & 146 & 10 & 156 \\
\hline Sergipe & 3 & 54 & 0 & 54 \\
\hline Região Sudeste & 92 & 3.382 & 20 & 3.402 \\
\hline Rio Grande do Sul & 38 & 619 & 5 & 624 \\
\hline Santa Catarina & 22 & 398 & 20 & 418 \\
\hline Região Centro-Oeste & 26 & 652 & 33 & 685 \\
\hline Distrito Federal & 1 & 175 & 10 & 185 \\
\hline Goiás & 5 & 159 & 0 & 159 \\
\hline Mato Grosso & 9 & 164 & 20 & 184 \\
\hline Mato Grosso do Sul & 11 & 154 & 3 & 157 \\
\hline
\end{tabular}

Fonte: Conselho Nacional de Secretários da Saúde, Leitos SRAG/UTI COVID-1912. 
A distribuição assimétrica dos leitos de UTI COVID-19 no Brasil pode ser melhor visualizada na Figura 1.

Figura 1 - Distribuição Espacial de Leitos habilitados SRAG/ UTI COVID-19. Brasil, 2020.

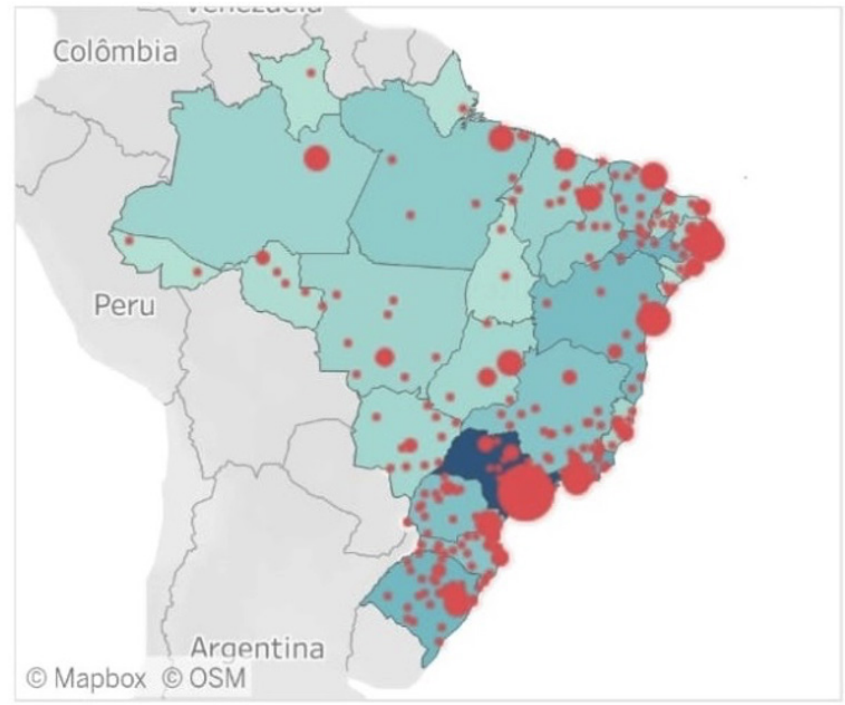

15

2.074

Fonte: Conselho Nacional de Secretários da Saúde, Leitos SRAG/UTI COVID-1912.

\section{DISCUSSÃO}

O início do surto de COVID-19 no Brasil fez emergir a preocupação como o provimento de Equipamentos de Proteção Individual (EPI) para suprir as equipes de saúde, disponibilidade de respiradores e de vagas em leitos de UTI em quantidades suficientes para atender a demanda gerada pela doença. O risco de um colapso no sistema de saúde brasileiro, a exemplo do vivenciado por outros países, tornou-se pauta recorrente na mídia, deixando os profissionais da saúde apreensivos e a população em pânico. Apesar de todo dos alertas, a rapidez com que a doença se disseminou em algumas regiões brasileiras confirmam os impactos da pandemia no sistema de saúde brasileiro, com real iminência de colapso ${ }^{8}$.

Os dados sobre a situação de leitos hospitalares, sobretudo os de UTI, mostram que o Brasil já apresentava um grande déficit que, do quadro epidemiológico da COVID-19, se acentuou, exigindo esforços dos gestores públicos nas três esferas de governo, para a ampliação de vagas, com o credenciamento de urgência de novos leitos. Além disso, a discrepância na distribuição dos leitos de UTI entre as diferentes regiões e estados brasileiros agrava ainda mais a situação do país para o enfrentamento da pandemia de COVID-19?.

Embora o Brasil possua uma proporção consolidada de leitos de UTI considerada satisfatória para os padrões esta- belecidos pela OMS e Ministério da Saúde (2,3 leitos/10 mil habitantes), a alocação desses leitos não acompanha a distribuição populacional ${ }^{13}$. A região Sudeste, considerada a mais populosa, concentra cerca de $42,0 \%$ da população brasileira e 50,2\% dos leitos de UTI disponíveis no território nacional, enquanto a região norte, que concentra $8,6 \%$ da população, conta com apenas 5,0\% dos leitos de UTI, tornando-se a região com menor disponibilidade desse tipo de leito ${ }^{11,14-15}$.

Esses dados são corroborados pelo Instituto de Estudos para Políticas de Saúde (IEPS), que mostra quase 15\% da população exclusivamente dependente do SUS não conta com leitos de UTI na região em que reside ${ }^{16}$. Além disso, a maioria dos leitos de UTI da rede pública pertence a grandes hospitais públicos, sobretudo de referência estadual ou regional, apresentando boa capacidade instalada e localizados quase sempre nas capitais ${ }^{11}$.

A distribuição da rede privada é guiada pelo mercado, sendo sensivel, portanto, à renda da população, diretamente relacionada à demanda por serviços de saúde pagos. Regiões com baixa densidade de leitos públicos de UTI também apresentam menor densidade de leitos privados, tornando improvável que a Saúde Suplementar atenda as demandas do SUS, apenas por meio da cessão de leitos excedentes durante a pandemia. Assim, a Fila Única de UTI é parte necessária da resposta à COVID-19, em situações de escassez de recursos, ainda que não dê conta de todas as iniquidades, devendo ser acompanhada também da ampliação do número de leitos nas regiões de maior carência e aumento proporcional do contingente de trabalhadores da saúde, e insumos necessários para operacionalizar o atendimento de alta complexidade.

A questão dos insumos, importante em todos os níveis de assistência, revela-se mais um gargalo na ampliação dos leitos de UTI. O Sistema Conselho Federal de Enfermagem (COFEN)/Conselhos Regionais de Enfermagem, que monitora a situação dos profissionais de Enfermagem desde o início da pandemia, registrou mais de 8.200 denúncias, a maior parte delas referentes à ausência ou inadequação de EPI e déficit de profissionais de Enfermagem/sobrecarga de trabalho ${ }^{17}$, evidenciando um iminente colapso do sistema de saúde, além do adoecimento e risco de morte dos profissionais da saúde que estão na linha de frente, atendendo as demandas pandêmicas $^{18}$.

Esse quadro reforça a importância da discussão sobre "Fila Única de UTI". Contudo, a utilização da capacidade ociosa da rede privada, conquanto proporcione alívio momentâneo, é insuficiente para fazer frente ao avanço da pandemia e ao colapso da assistência, como visto em Manaus. A questão que enfrentamos é: Quem se deixará morrer sem a assistência necessária? Quem passará na frente dos que esperam acesso a 
um leito UTI, tendo atendimento prioritário, independente do quadro clínico?

Não se trata de um cenário futuro hipotético. Dados da AMIB indicam que, entre 1 으 de março e 15 de maio, pacientes internados em UTI COVID-19 de hospitais públicos tiveram quase o dobro de mortalidade do que em hospitais privados. Analisando 13.941 pacientes com o novo coronavírus que fizeram tratamento, a taxa de mortalidade nas unidades privadas ficou em $19,5 \%$, enquanto em hospitais públicos chegou a 38,5\%. A discrepância indica um atendimento tardio, mormente quando se considera o quantitativo de pacientes que necessitaram de ventilação mecânica, importante indicativo de gravidade do quadro. Dos internados em leitos particulares, $36,8 \%$ precisaram de ventilação, enquanto na rede pública o número chegou a 66,5\%. Quando se considerada apenas os pacientes com necessidade de ventilação mecânica, a mortalidade da rede pública e privada se aproxima, sendo registrado o índice de $70,5 \%$ nos hospitais públicos e $63,6 \%$ nos privados ${ }^{19}$

A proposta de "Fila Única", longe de ser uma aventura radical, encontra respaldo em bem-sucedidas experiências, como a "Fila Única do Transplante", que tornou o Brasil uma referência internacional no transplante de órgãos. Já existe, também, arcabouço jurídico para a implementação a Lei no 13.979 , de 6 de fevereiro de 2020, que “dispõe sobre as medidas para enfrentamento da emergência de saúde pública de importância internacional decorrente do coronavírus responsável pelo surto de 2019", e autoriza formas de requisição pública de bens e serviços para enfrentamento à pandemia ${ }^{20}$.

O aumento expressivo do número de casos de COVID-19 no país torna necessário e urgente racionalizar o uso dos equipamentos de saúde. Nesse contexto, a criação de uma "Fila Única" nas UTI, como defendido pelo Conselho Nacional de Saúde (CNS), que sugere "aos gestores do SUS, em seu âmbito de competência, que requisitem leitos privados, quando necessário, e procedam à sua regulação única a fim de garantir atendimento igualitário durante a pandemia"21.

Essa medida pode assegurar o acesso mais equitativo ao atendimento, ajudando a salvar vidas, em um contexto de sobrecarga do Sistema de Saúde. A distribuição racional dos recursos hospitalares de alta complexidade pode reduzir a mortalidade em decorrência das complicações pela COVID-19, especialmente quando se considera o elevado tempo de duração das hospitalizações e internações em UTI, em relação a outras sindromes respiratórias virais, e a baixa adesão ao distanciamento social, que poderia desacelerar o ritmo de transmissão e, consequentemente, a demanda por atendimentos de alta complexidade, por respiradores, ventiladores pulmonares e leitos de UTI.
Medidas de pouco alcance já vêm sendo adotadas por alguns gestores, a exemplo do Ceará, que passou a utilizar um hospital privado que encontrava-se fechado há duas décadas. Ainda, acordos com hospitais privados aumentaram o número de leitos em estados como Pernambuco e São Paulo, onde o Hospital da Cruz Vermelha e o da Universidade Santo Amaro (UNISA) estão recebendo pacientes do SUS e recebendo remuneração pela tabela SUS $22-25$.

No entanto, a alta velocidade de propagação do SARS-CoV-2 na população e o atual contexto epidemiológico da pandemia exigem um melhor ordenamento da regulação e do gerenciamento dos leitos de alta complexidade. Embora os hospitais tenham critérios para a distribuição de vagas na UTI, a centralização da decisão de uso dos leitos de UTI em momentos de escassez de recursos, pode ser uma alternativa válida e eficaz. Assim, a administração federal dos leitos de UTI privados disponíveis, de forma colaborativa ou compulsória, com adoção de critérios homogêneos, permitiria uma distribuição equânime dos leitos ${ }^{26}$

Para tanto, a alocação justa dos leitos requer uma estrutura ética, critérios técnicos e científicos consolidados e aspectos jurídicos legais, respeitando-se a dignidade humana de todos os indivíduos. Além disso, as decisões não podem recair sobre os profissionais da linha de frente, evitando-se a intensificação de falhas, estresse e adoecimento, e preservando a integridade destes trabalhadores, imprescindiveis para o enfrentamento deste quadro epidemiológico ${ }^{27}$.

Assim, entende-se que o momento de pandemia exige que os gestores incluam, em sua pauta, diretrizes para o alcance dos princípios doutrinários do SUS: a "universalidade", garantindo acesso de todos os brasileiros aos bens e serviços de saúde, sobretudo leitos de terapia intensiva; a "integralidade", assegurando ações e serviços de saúde nos diferentes pontos da RAS; e a "equidade", priorizando o atendimento para os de maior vulnerabilidade social e sanitária, sem perder de vista os determinantes sociais.

Para tanto, os valores éticos que devem direcionar a alocação de recursos durante a pandemia de COVID-19 são: maximização de benefícios; priorização dos profissionais da saúde; não alocação por ordem de chegada; sensibilidae às evidências; reconhecimento da participação na pesquisa; e aplicação dos mesmos princípios a todos os pacientes $\mathrm{CO}$ VID-19 e não-COVID-1928

\section{Limitações do Estudo}

A principal limitação deste estudo é a incerteza sobre o comportamento do novo coronavírus no Brasil, nos próximos meses, o que traz significativo impacto na análise e prospecção de cenários. 


\section{Contribuições do Estudo para a Prática}

O estudo contribui com informações relevantes sobre a distribuição e oferta de leitos de UTI gerais e específicos para COVID-19 no Brasil, instigando a reflexão sobre a necessidade e relevância de uma "fila única de leitos", gerenciada pelos gestores do Sistema Único de Saúde (SUS), nas diferentes esferas de governo.

\section{CONSIDERAÇÕES FINAIS}

O enfrentamento da pandemia de COVID-19 tem apontado diversos desafios para os governos de Nações do mundo todo. No caso do Brasil, que possui um importante e grandioso sistema de saúde como o SUS, os gestores dos diferentes níveis federativos são desafiados a garantir acesso aos serviços de saúde a todos os doentes, especialmente aqueles críticos, com complicações e/ou com comorbidades (a exemplo problemas cardiacos, diabetes e obesidade), que evoluam para um quadro clínico respiratório grave e necessitem de cuidados intensivos.

A "Fila Única" de leitos de UTI (públicos e privados), coor- denada pelos gestores do SUS, nas diferentes esferas de governo, é uma alternativa que pode ser eficaz para otimizar leitos e ventiladores pulmonares, garantir equipamentos, insumos estratégicos, medicações de alto custo e profissionais qualificados para o manejo clínico dos pacientes críticos.

Para isso, é necessário a criação de um painel atualizado de UTI, com regulação pelas três esferas de governo, com o intuito de manter a autonomia dos entes federados, contendo: o número de leitos gerais e específicos para COVID-19; taxa de ocupação diária; disponibilidade diária de leitos; equipamentos existentes; lista de insumos hospitalares, como os produtos farmacêuticos; EPI; quantidade e qualificação dos profissionais existentes na equipe; fila de espera, dentre outros, para salvar centenas, ou talvez milhares de vidas. Pois, "Cada vida importa" sim!

Contribuição dos Autores: MCM Fagundes, NP Freire, MH Machado e FRG Ximenes Neto participaram da concepção e delineamento do estudo, redação e revisão do conteúdo intelectual até a versão final do manuscrito.

\section{REFERÊNCIAS}

1. World Health Organization (WHO). Emergencies preparedness, response: Novel Coronavirus - China Disease outbreak news: Update. [Internet]. 2020 [cited $2020 \mathrm{abr}$ 26]. Available from: https://www.who.int/csr/ don/12-january-2020-novel-coronavirus-china/en/.

2. Huang C, Wang Y, Li X, Ren L, Zhao J, Hu Yi et al. Clinical features of patients infected with 2019 novel coronavirus in Wuhan, China. The Lancet. [Internet]. 2020 [cited 2020 abr 26]; 395(10223):497-506. Available from: https:// www.thelancet.com/journals/lancet/article/PIISO1406736(20)30183-5/fulltext.

3. Wang C, Horby PW, Hayden FG, Gao GF. A novel coronavirus outbreak of globalhealth concern. The Lancet. [Internet]. 2020 [cited 2020 abr 26]; 395(10223):470-473. Available from: https://www.thelancet.com/journals/ lancet/article/PIIS0140-6736(20)30185-9/fulltext.

4. World Health Organization (WHO). WHO DirectorGeneral's statement on IHR Emergency Committee on Novel Coronavirus (2019-nCoV). [Internet]. 2020 [cited $2020 \mathrm{abr}$ 26]. Available from: https://www.who.int/dg/ speeches/detail/who-director-general-s-statementon-ihr-emergency-committee-on-novel-coronavirus(2019-ncov)

5. World health organization (WHO). WHO DirectorGeneral's opening remarks at the media briefing on COVID-19. [Internet]. 2020 [cited 2020 Apr 26]. Available from: https://www.who.int/dg/speeches/detail/whodirector-general-s-opening-remarks-at-the-mediabriefing-on-covid-19---11-march-2020.
6. Ministério da Saúde (BR). Brasil registra primeiro caso da doença. [Internet] 2020 Feb 26 [cited 2020 Apr 26]. Available from: https://www.saude.gov.br/noticias/ agencia-saude/46771-coronavirus-brasil-registra-49492-casos-e-3-313-mortes.

7. Ministério da Saúde (BR). Resolução n 466/2012, de 12 de dezembro de 2012. Discorre sobre as Diretrizes e Normas Regulamentadoras de pesquisa envolvendo seres humanos [Internet]. 2012. [cited 2020 June 12]. Available from: http://conselho.saude.gov.br/resolucoes/2012/ Reso466.pdf.

8. Ministério da Saúde (BR). Coronavirus - Brasil. [Internet] 2020 [cited 2020 June 16]. Available from: https://covid.saude.gov.br/

9. Fundação Osvaldo Cruz (Fiocruz). MonitoraCovid-19: Interiorização do Covid-19 e as redes de atendimento em saúde. ICICT-FIOCRUZ. [Internet]. 2020 [cited 2020 June 17]. Available from: https://portal.fiocruz.br/sites/ portal.fiocruz.br/files/documentos/monitoracovid_ notatecnica_04_05_20.pdf.

10. Instituto Brasileiro de Geografia e Estatistica (IBGE). Informações de saúde: subsídios ao enfrentamento regional à COVID-19 - Nota Técnica 01/2020. [Internet]. 2020 [cited 2020 May 7]. Available from: https://leitosibgedgc.hub.arcgis.com/.

11. Ministério da Saúde (BR). Painel de leitos e insumos. [Internet]. 2020 [cited 2020 June 8]. Available from: https://covid-insumos.saude.gov.br/paineis/insumos/ painel_leitos.php. 
12. Conselho Nacional de Secretários da Saúde (CONASS). Leitos SRAG/UTI COVID-19 - Monitoramento de Habilitações. [Internet]. 2020 [cited 2020 June 26]. Available from: http://www.conass.org.br/leitos-sraguti-covid-19-monitoramento-de-habilitacoes/.

13. Associação de Medicina Intensiva Brasileira. AMIB apresenta dados atualizados sobre leitos de UTI no Brasil. [Internet]. 2020 [cited 2020 June 26]. Available from: http://www.epsjv.fiocruz.br/sites/default/files/ files/dados_uti_amib(1).pdf.

14. Ministério da Saúde (BR). Instituto Nacional do Câncer. Projeção populacional para o ano de 2020 por Unidade da Federação, Capital e Brasil [Internet]. 2020 [cited 2020 June 27]. Available from: https://www.inca.gov.br/ estimativa/anexo-c-projecao-populacional-para-o-anode-2020-por-unidade-da-federacao-capital-e-brasil.

15. Instituto Brasileiro de Geografia e Estatística (BR). Sinopse do Censo Demográfico 2010 [Internet]. 2020 [cited 2020 June 27]. Available from: https://censo2010. ibge.gov.br/sinopse/index.php?uf=00\&dados=1.

16. Rache B, Rocha R, Nunes L, Spinola P, Massuda A. Para Além do Custeio: Necessidades de Investimento em Leitos de UTI no SUS sob Diferentes Cenários da COVID-19. São Paulo: Instituto de Estudos para Politicas de Saúde (IEPS). [Internet]. 2020 [cited 2020 June 26]. Available from: https://ieps.org.br/wp-content/ uploads/2020/04/IEPS-NT7.pdf.

17. Conselho Federal de Enfermagem (COFEN). Observatório de Enfermagem. Profissionais infectados com COVID-19 informado pelos enfermeiros responsáveis técnicos/coordenadores. [Internet] 2020 [cited 2020 May 5]. Available from: http:// observatoriodaenfermagem.cofen.gov.br/.

18. Lourenção LG. A Covid-19 e os desafios para o sistema e os profissionais de saúde. Enferm Foco. [Internet]. 2020 [cited 2020 June 27]; 11(1):6-7. Available from: http:// revista.cofen.gov.br/index.php/enfermagem/article/ view/3488

19. Associação de Medicina Intensiva Brasileira (AMIB). UTI brasileiras: Registro nacional de terapia intensiva. [Internet] 2020 [cited 2020 June 27]. Available from: http://www.utisbrasileiras.com.br/sari-covid-19/ benchmarking-covid-19/.

20. Brasil. Lei no 13.979, de 6 de fevereiro de 2020 - Dispõe sobre as medidas para enfrentamento da emergência de saúde pública de importância internacional decorrente do coronavirus responsável pelo surto de 2019. DOU [Internet]. $2020 \mathrm{Feb} 07$ [cited 2020 June 26]; 27(1):1. Available from: http://www.in.gov.br/en/web/dou/-/lein-13.979-de-6-de-fevereiro-de-2020-242078735.

21. Conselho Nacional de Saúde (BR). Recomendação noㅡ 026, de 22 de abril de 2020 - Recomenda aos gestores do SUS, em seu âmbito de competência, que requisitem leitos privados, quando necessário, e procedam à sua regulação única a fim de garantir atendimento igualitário durante a pandemia. [Internet]. 2020 [cited 2020 June 26]. Available from: http://www.susconecta.org.br/wpcontent/uploads/2020/04/Reco026-Aos-gestoresRequisi\%C3\%A7\%C3\%A30-de-leitos-privados-eregula\%C3\%A7\%C3\%A30-\%C3\%BAnica_Covid-19.pdf.

22. Associação de Medicina Intensiva Brasileira (BR). Pesquisa AMIBnet apurou o cenário das UTI Brasileiras para enfrentamento da Covid-19 - Dados foram coletados em abril e avaliou dados de UTIs Brasileiras. [Internet] 2020 [cited 2020 May 21]. Available from: https://www. amib.org.br/noticia/nid/pesquisa-amibnet-apurou-ocenario-das-utis-brasileiras-para-enfrentamento-dacovid-19/.

23. Bruno M. Fortaleza lidera taxa de novos casos de coronavirus nas capitais e Ceará já requisita leitos de hospitais privados. El Pais [Internet] $2020 \mathrm{Apr} 8$ [cited 2020 June 26]; Available from: https://brasil.elpais. com/sociedade/2020-04-09/fortaleza-lidera-taxa-denovos-casos-de-coronavirus-nas-capitais-e-ceara-jarequisita-leitos-de-hospitais-privados.html.

24. Cruz EP. Covas fecha parceria com 11 hospitais privados para alugar leitos - Cada leito custará R\$2,1 mil por dia de ocupação. Agência Brasil [Internet] 2020 Apr 8 [cited 2020 May 8]. Available from: https://agenciabrasil. ebc.com.br/saude/noticia/2020-05/covas-fechaparceria-com-1l-hospitais-privados-para-alugar-leitos.

25. Noronha KVMS, Guedes GR, Turra CM, Andrade MV, Botega L, Nogueira D et al. Pandemia por COVID-19 no Brasil: análise da demanda e da oferta de leitos hospitalares e equipamentos de ventilação assistida segundo diferentes cenários. Cad. Saúde Pública [Internet]. 2020 [cited 2020 July 05]; 36(6):e00115320. Available from: https://doi.org/10.1590/0102$311 \times 00115320$.

26. Moreira RS. COVID-19: unidades de terapia intensiva, ventiladores mecânicos e perfis latentes de mortalidade associados à letalidade no Brasil. Cad. Saúde Pública [Internet]. 2020 [cited 2020 July 04]; 36(5):e00080020. Available from: http://dx.doi.org/10.1590/0102-311x00080020.

27. Satomi E, Souza PMR, Thomé BC, Reingenheim C, Werebe E, Troster EJ et al. Fair allocation of scarce medical resources during COVID-19 pandemic: ethical considerations. Einstein (São Paulo) [Internet]. 2020 [cited 2020 July 04]; 18:eAE5775. Available from: https://doi.org/10.31744/einstein_journal/2020ae5775.

28. Emanuel EJ, Persad G, Upshur R, Thome B, Parker M, Clickman A. Fair Allocation of Scarce Medical Resources in the Time of Covid-19. N Engl J Med. [Internet]. 2020 [cited 2020 Jun 27]; 382(21):2049-2055. Available from: https://www.nejm.org/doi/full/10.1056/nejmsb2005114. 